\title{
A sample of galaxy clusters to study the fundamental plane: Redshift measurements ${ }^{\star}$
}

\author{
S. Maurogordato ${ }^{1}$, D. Proust ${ }^{1}$, A. Cappi ${ }^{2}$, E. Slezak ${ }^{3}$, and J.M. Martin ${ }^{4}$ \\ 1 DAEC, CNRS, Observatoire de Paris-Meudon, 5 place J. Janssen, 92195 Meudon, France \\ e-mail: maurogordato@obspm.fr \\ 2 Osservatorio Astronomico di Bologna, via Zamboni 33, I-40126 Bologna, Italy \\ e-mail: cappi@astbo3.bo.astro.it \\ 3 Observatoire de Nice, B4229, Le Mont-Gros, 06304 Nice Cedex 4, France \\ e-mail: slezak@obs-nice.fr \\ 4 ARPEGES, Observatoire de Paris-Meudon, 5 place J. Janssen, 92195 Meudon, France \\ e-mail: jmmartin@obspm.fr
}

Received June 24; accepted October 7, 1996

\begin{abstract}
We present the results of spectroscopic observations of galaxies in a sample of nearby clusters. We measured galaxy redshifts and estimated cluster velocity dispersions. These observations were carried out at the Observatoire de Haute Provence as part of a program aiming at a more detailed study of the Cluster Fundamental Plane.
\end{abstract}

Key words: galaxies: clusters of; redshifts - Cosmology: observations

\section{Introduction}

Clusters of galaxies are the largest gravitationally bound systems, with three components contributing to their total mass: the dark matter (the dominant one), the hot X-ray emitting gas (the dominant baryonic component), and the optically luminous stars in galaxies. Determining the distribution of these three components and their correlations represents a key problem in modern cosmology.

A possible approach is offered by studying the relations between those global cluster properties that can be more or less directly and easily measured, such as the velocity dispersion $\sigma$, the total luminosity $L$, and the effective radius $R_{\mathrm{e}}$. Correlations between intrinsic parameters have effectively been found for galaxy clusters, as for instance between richness and velocity dispersion (Danese et al. 1980; Cole 1989), and between radius

Send offprint requests to: S. Maurogordato

* Based on observations made at Haute-Provence Observatory, CNRS, France. and luminosity (West et al. 1989). However these relations have a high dispersion. Schaeffer, et al. (1993, hereafter SMCB) analysed a sample of 19 clusters for which luminosities, radii (West et al. 1989) and velocity dispersions (Struble \& Rood 1991) were available. In addition to the radius-luminosity relation found by West et al. (1989), $R \propto L^{0.5 \pm 0.1}, \mathrm{SMCB}$ have also defined a luminosity-velocity dispersion relation, $L \propto \sigma^{2.0 \pm 0.4}$ (much better than the old richness-velocity dispersion relation). By analogy with elliptical galaxies, for which the above three parameters can be combined in one relation (Dressler et al. 1987; Djorgovski \& Davis 1987) defining the so-called "fundamental plane", and given that virialized systems should show a relation between mass, radius and velocity dispersion, SMCB have minimized the relation $L \propto R^{\alpha} \sigma^{\beta}$, and found a best fit with a considerably lower dispersion than the one-independent parameter relations. The parameters $\alpha=0.89 \pm 0.15$ and $\beta=1.28 \pm 0.10$ have values similar to those measured for the fundamental plane of elliptical galaxies.

The number of clusters for which the three parameters $L, R$ and $\sigma$ are reliably measured is however small. Therefore we have started an observational program to increase the cluster database, a necessary condition for further studies of the Cluster Fundamental Plane. We present in this paper the results of our spectroscopic observations of a sample of nearby clusters (redshifts of member galaxies and preliminary estimates of velocity dispersions).

\section{The sample}

Targets were selected in order to cover a large spread of masses (richness class 1 to 4 ) and redshifts (0.04 to 0.25 ): they constitute a sample of 16 Abell/ACO clusters. 
Sufficiently nearby clusters, i.e. A195, A1035, A1045, A1126, A1413, A1468, A1781, A1831, A2034, A2245, A2312, A2457 have been observed at the Observatoire de Haute-Provence, and the results are presented in this paper. The farthest ones, A222, A223, A520, A521, have been observed using the capabilities for multi-object spectroscopy at CFHT and ESO and will be analysed in a separate paper.

The sample was a priori composed of relatively regular clusters, in order to derive "unambiguous" velocity dispersions. Nevertheless, in a few cases the velocity distribution (cf. Sect. 4) has shown evidence of subclustering, projection effects or bimodality, which obviously affect the estimate of the velocity dispersion. For this reason the three clusters A1035, A1781, A1831 with a complex velocity distribution will not be included in future in our analysis.

In addition to the spectroscopic data, we have also obtained photometric measurements based on automatic scans of POSSII plates, which will be presented in a forthcoming paper.

\section{Observations and data reduction}

A catalogue of galaxies up to magnitude $m_{\mathrm{R}}=18.5$ has been built applying the algorithms described in Slezak et al. (1988) to automatic scans of POSSII plates, thanks to the MAMA facilities; this catalogue included the coordinates of galaxies within a radius of one degree from each cluster centre. Selected galaxies have been observed at the $193 \mathrm{~cm}$ telescope of the Observatoire de HauteProvence, using the Carelec spectrograph in long slit mode (Lemaitre et al. 1990). The choice of the grating represented a compromise between good resolution and reasonable exposure time, and was also constrained by the wavelength range suited for line identification at low-redshift $(\sim 3600-7300 \AA)$. The dispersion was $260 \AA / \mathrm{mm}$, corresponding to $\sim 7 \AA /$ pixel with the CCD TK512 $(512 \times 512$ pixels of $27 \mu \mathrm{m}$ ), and with the slit aperture of $1^{\prime \prime}$ the spectral resolution was $8 \AA$. The following results are based on four observing runs (7 nights each one) in April and September 1993, and in April and May 1994. Unfortunately more than half of the nights were lost for bad weather conditions.

The exposure time was 45 minutes; two exposures were taken for faint objects. Wavelength calibrations were done using an He lamp and an Ar lamp before each exposure. Standard stars were observed each night (HD 102494, HD 171232, HD 112299, HD 140913). We obtained the spectra of about $8 / 10$ objects per night. Usually we obtained one spectrum per exposure, but in some cases, for instance when sampling the core of rich clusters, we rotated the spectrograph in order to get simultaneously the spectra of a few objects. At the end, we have collected 93 spectra with a sufficient signal-to-noise ratio to allow a good redshift determination. Data reduction was carried out with IRAF, using the MULTIRED package. MULTIRED is an integrated multi-slit spectra reduc- tion task (Le Fèvre et al. 1995) which allows to process raw 2D spectra CCD frames (with one or several spectra) into bias/flat/sky corrected 2D spectra and wavelength calibrated $1 \mathrm{D}$ spectra. Radial velocities have been determined using the cross-correlation technique (Tonry \& Davis 1979) implemented in the RVSAO package (developed at the Smithsonian Astrophysical Observatory).

Finding charts for each cluster are displayed in Fig. 1. They cover $15^{\prime} \times 15^{\prime}$ square regions extracted from the Space Telescope Science Institute Digital Sky Survey ${ }^{1}$ around the position of the cluster in the Abell/ACO catalogue $\left(30^{\prime} \times 30^{\prime}\right.$ for A1413, A1781 and A2457). An identifying number is displayed to the right of each target.

Velocity measurements are listed in Table 1. The columns are as follows:

Column (1): Abell cluster number (Abell et al. 1989); Column (2): identification number of each target galaxy in the cluster as shown in the finding charts; Columns (3) and (4): right ascension and declination (J2000.0) of the target galaxy; Column (5): best estimate of the radial velocity resulting from the cross-correlation technique; Column (6): estimated error; Column (7): other published measurement of the radial velocity. The subscript $^{e}$ indicates that the measurement refers to the emission line velocity.

\section{Velocity analysis of the clusters}

The number of measured redshifts per cluster is often quite small (from 5 to 20 galaxies). It is therefore difficult to have reliable estimates of the velocity dispersion. Using the NED database ${ }^{2}$ we searched for other galaxies with measured redshifts within a radius of one degree from the center of each target cluster. This allowed us to add one more velocity to A1035 (Batuski et al. 1991), three to A1413 (Stocke et al. 1991; Allen et al. 1992), one to A1045 (Allen et al. 1992), sixteen to A1831 (NED 1992 and Owen et al. 1995), two to A2034 (Crawford et al. 1995), four to A195 (Giovanelli \& Haynes 1993), four to A2245 (from Rhee \& Katgert (1988) and NED 1992), six to A 2457

1 Based on photographic data of the National Geographic Society - Palomar Observatory Sky Survey (NGS-POSS) obtained using the Oschin Telescope Palomar Mountain. The NGS-POSS was funded by a grant from the National Geographic Society to the California Institute of Technology. The plates were processed into the present compressed digital form with their permission. The Digitized Sky Survey was produced at the Space Telescope Science Institute under US Government grant NAG W-2166.

Copyright (C)1994, Association of Universities for Research in Astronomy, Inc. All rights reserved.

2 The NASA/IPAC Extragalactic Database (NED) is operated by the Jet Propulsion Laboratory, California Institute of Technology, under contract with the National Aeronautics and Space Administration. 


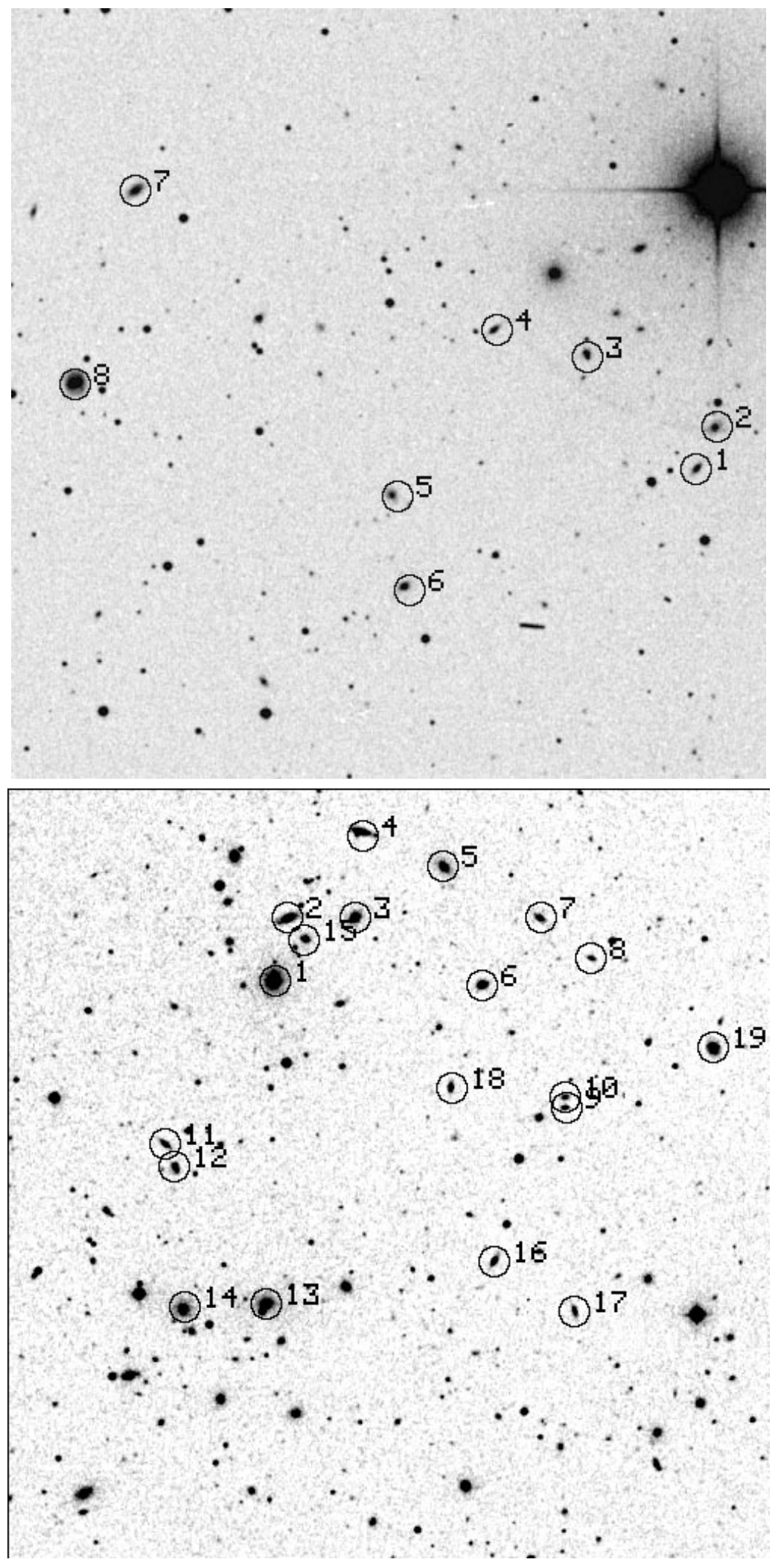

Fig. 1. a) to 1): Finding charts for our nearby sample of clusters: a) A195, b) A1035, c) A1045, d) A1126, e) A1413, f) A1468, g) A1781, h) A1831, i) A2034, j) A2245, k) A2312, l) A2457. These frames are extracted from the Digital Sky Survey 


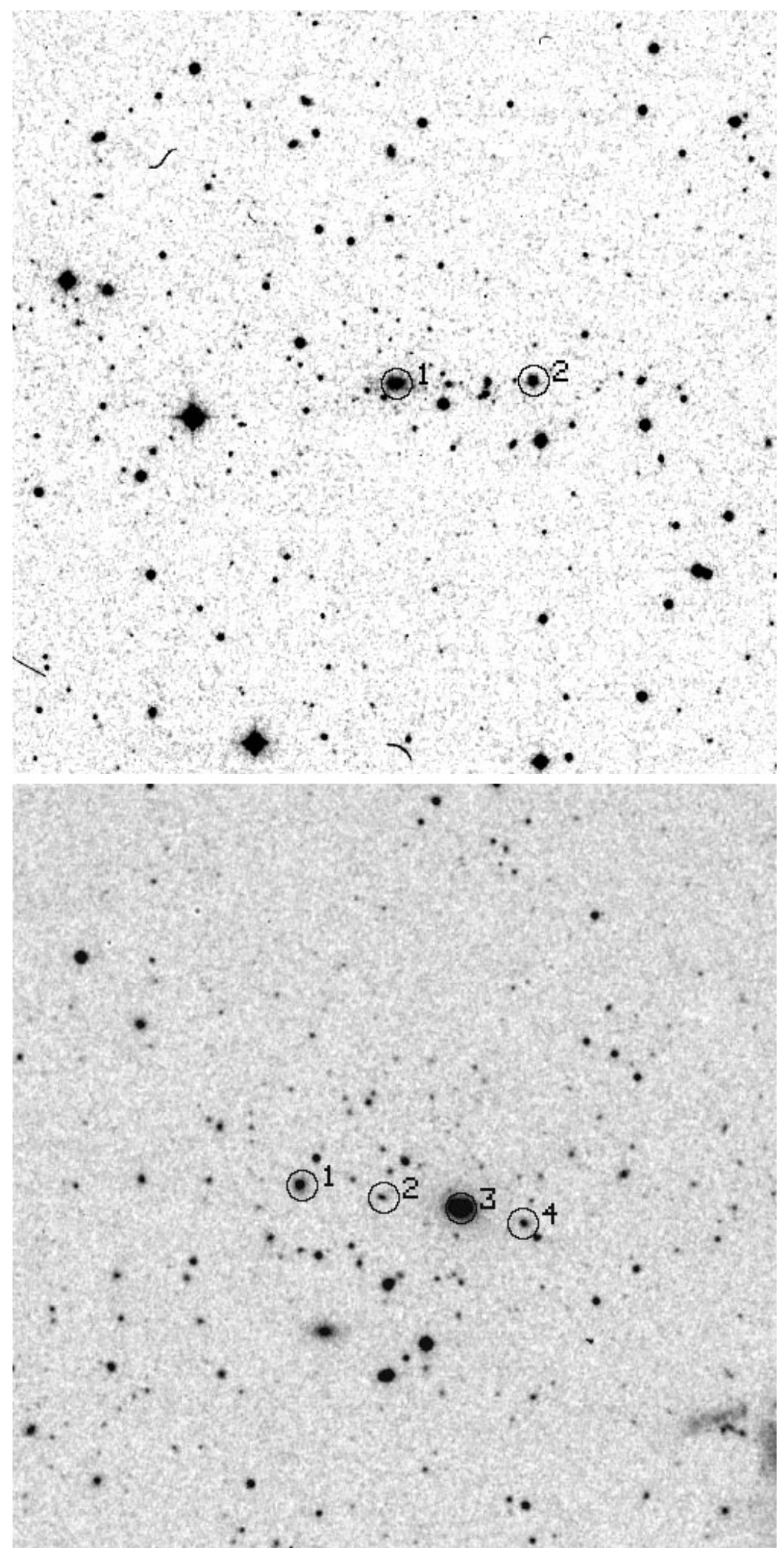

Fig. 1. c-d) continued 


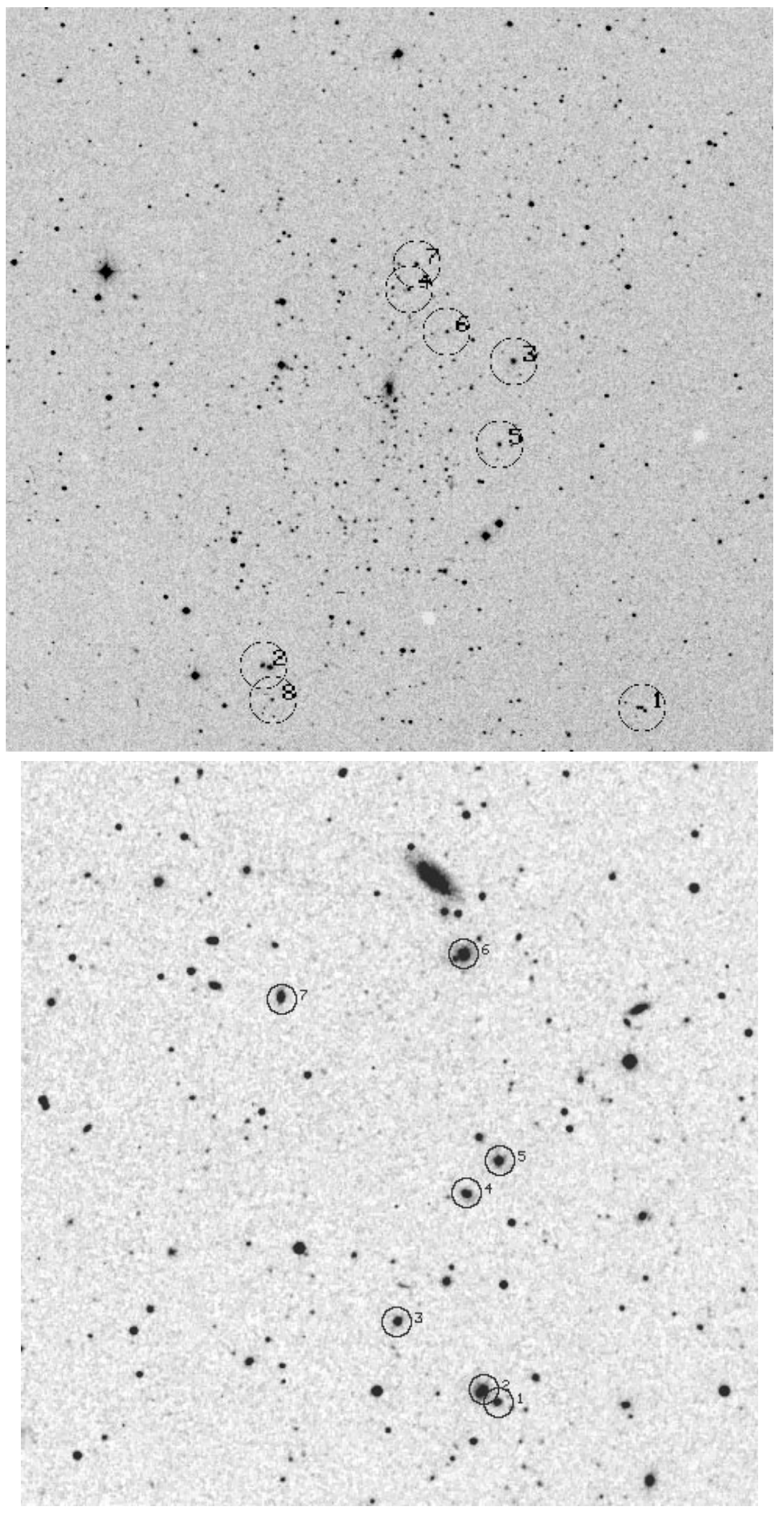

Fig. 1. e-f) continued 


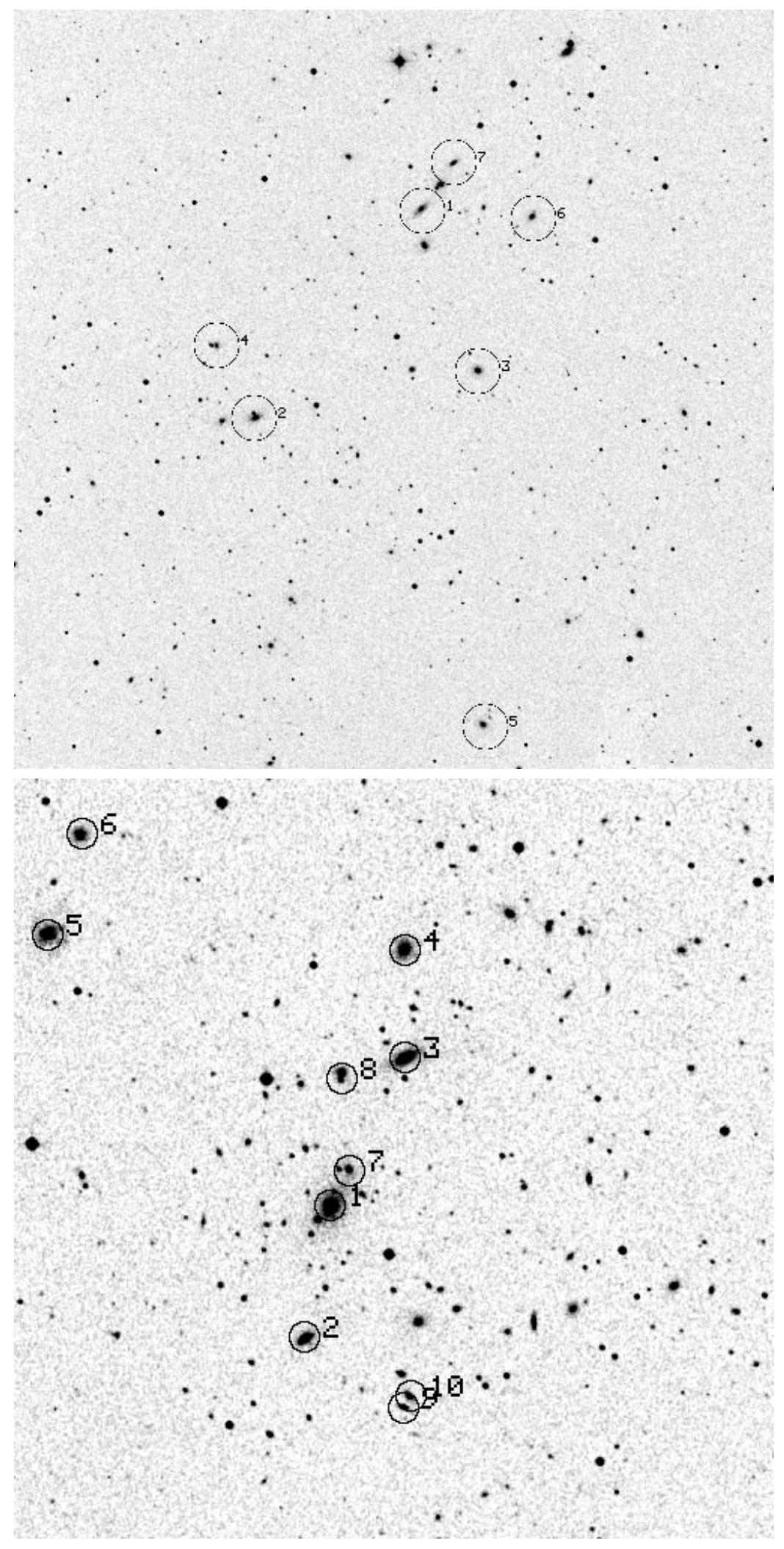

Fig. 1. g-h) continued 


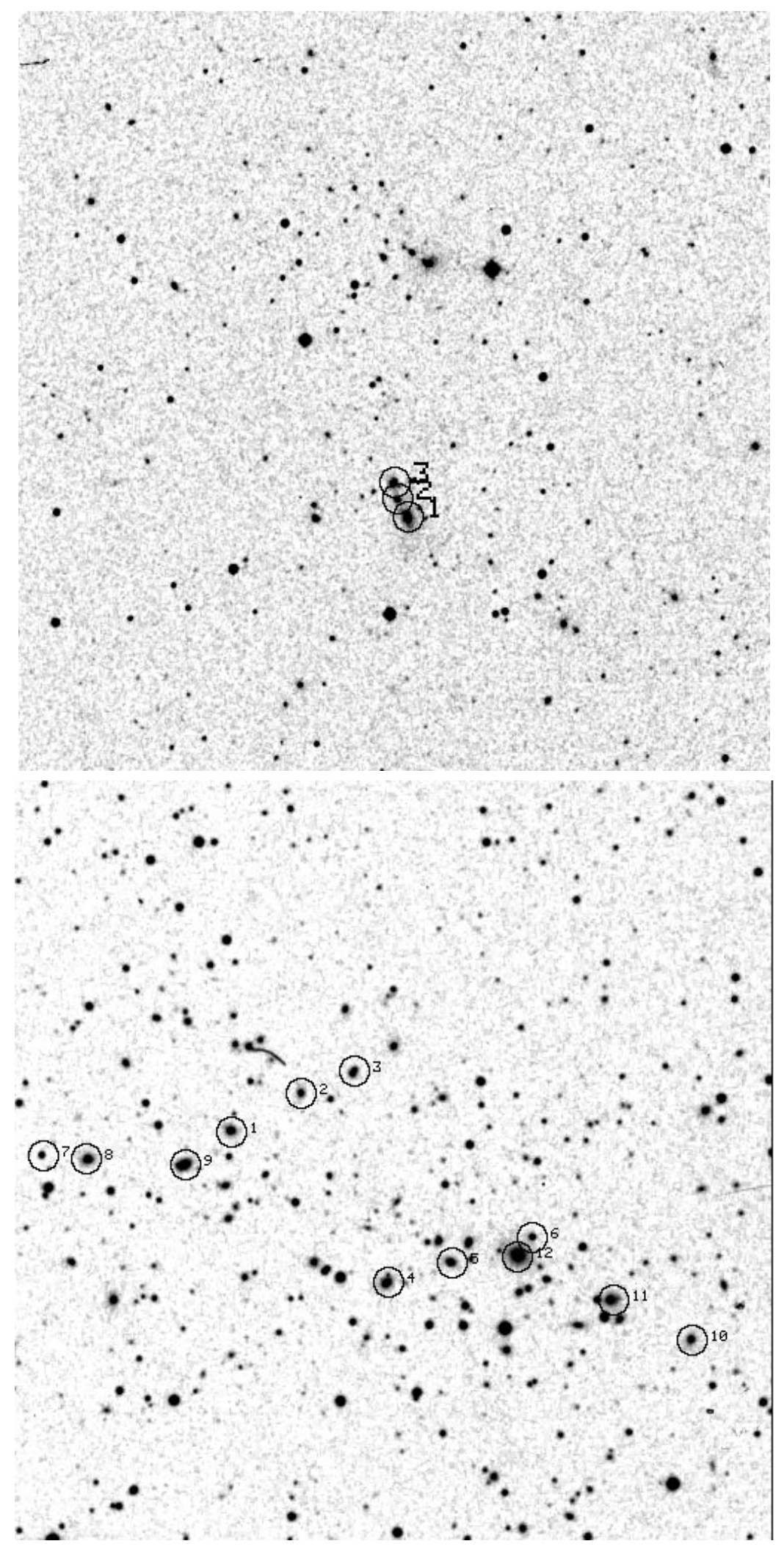

Fig. 1. i-j) continued 


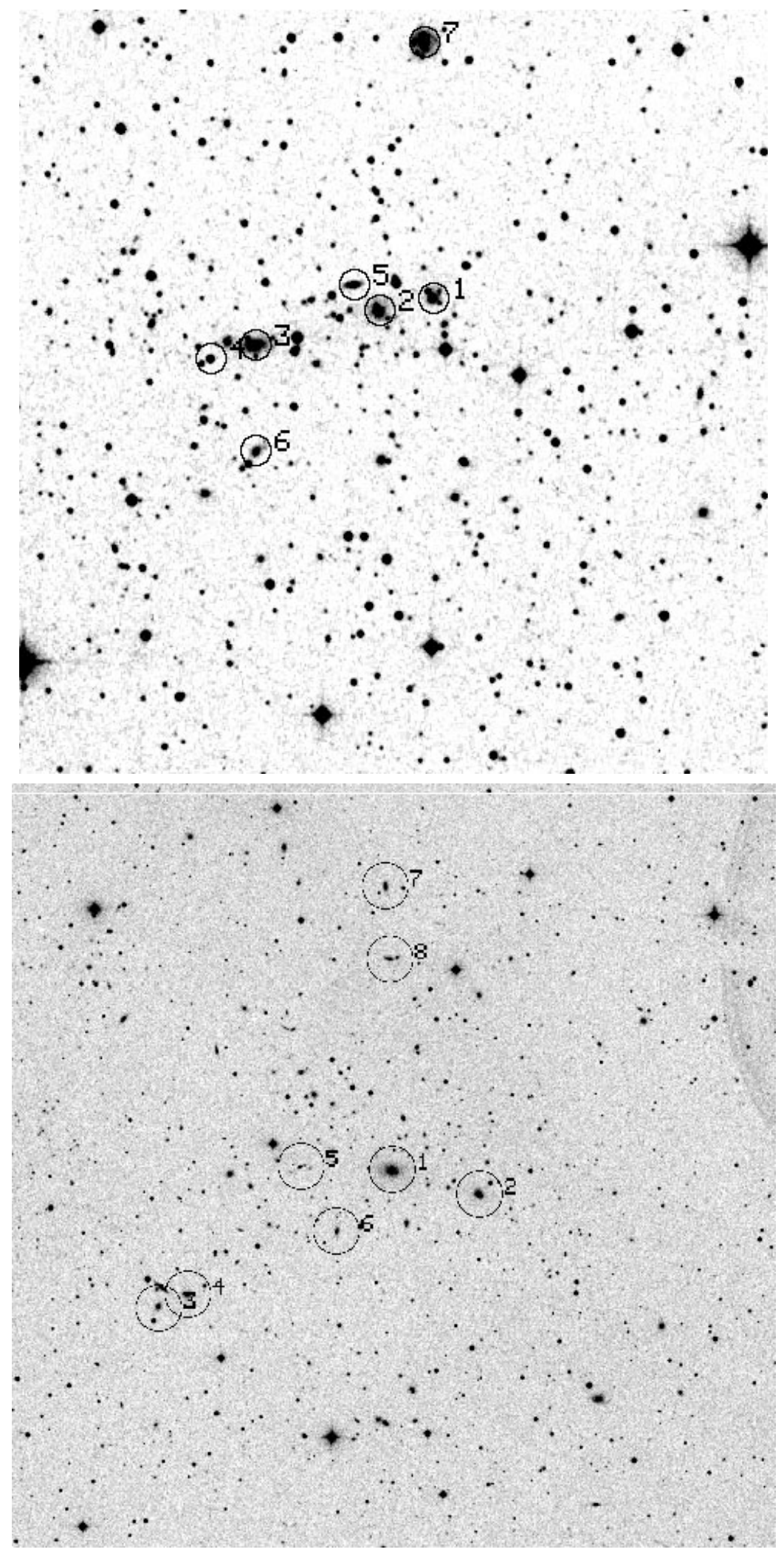

Fig. 1. k-l) continued 
Table 1. Heliocentric redshifts for galaxies

\begin{tabular}{|c|c|c|c|c|c|c|}
\hline CLUSTER & NUMBER & $\begin{array}{l}\text { R.A. } \\
(2000)\end{array}$ & $\begin{array}{l}\text { DEC. } \\
(2000)\end{array}$ & $\begin{array}{l}\text { HEL. VEL. } \\
V\left(\mathrm{~km} \mathrm{~s}^{-1}\right)\end{array}$ & $\begin{array}{l}\text { ERROR } \\
\Delta V\left(\mathrm{~km} \mathrm{~s}^{-1}\right)\end{array}$ & $\begin{array}{l}\text { Other measurements } \\
V\left(\mathrm{~km} \mathrm{~s}^{-1}\right)\end{array}$ \\
\hline \multirow[t]{9}{*}{$\overline{\mathrm{A} 195}$} & 1 & 012643.0 & 190913.4 & 12464 & 37 & \\
\hline & 2 & 012641.6 & 191001.2 & 12369 & 34 & \\
\hline & 3 & 012651.8 & 191123.1 & 12318 & 52 & \\
\hline & 4 & 012659.2 & 191149.2 & & & \\
\hline & 5 & 012707.0 & 190839.3 & 12626 & 41 & \\
\hline & 6 & 012706.2 & 190657.4 & 12924 & 58 & \\
\hline & 7 & 012647.6 & 191319.3 & 12682 & 51 & \\
\hline & 8 & $\begin{array}{lll}01 & 27 & 27.9\end{array}$ & 191419.5 & 14066 & 65 & \\
\hline & 9 & 012732.5 & 191042.8 & 5212 & 29 & \\
\hline \multirow[t]{19}{*}{$\overline{\mathrm{A} 1035}$} & 1 & 103214.0 & 401615.2 & 23261 & 35 & \\
\hline & 2 & 103212.6 & 401726.4 & 23869 & 43 & \\
\hline & 3 & 103205.9 & 401725.0 & 21714 & 35 & \\
\hline & 4 & 103205.0 & 401903.5 & 13025 & 95 & \\
\hline & 5 & 103156.9 & 401821.0 & 23669 & 51 & \\
\hline & 6 & 103153.5 & $4016 \quad 07.7$ & 20761 & 74 & \\
\hline & 7 & 103147.6 & $4017 \quad 19.5$ & 22222 & 78 & \\
\hline & 8 & 103142.5 & 401634.2 & 24436 & 49 & \\
\hline & 9 & 103145.7 & 401346.6 & 19874 & 40 & \\
\hline & 10 & 103145.4 & 401358.4 & 22801 & 69 & \\
\hline & 11 & 103224.8 & $4013 \quad 12.0$ & 20169 & 59 & \\
\hline & 12 & 103224.0 & 401248.0 & 23939 & 16 & \\
\hline & 13 & 103215.2 & 401009.8 & 20054 & 49 & \\
\hline & 14 & 103223.3 & $4010 \quad 09.7$ & 20092 & 33 & \\
\hline & 15 & 103211.0 & 401700.5 & 24460 & 41 & \\
\hline & 16 & 103152.8 & 401056.3 & 20438 & 78 & \\
\hline & 17 & 103144.9 & 400958.5 & 19799 & 97 & \\
\hline & 18 & 103156.7 & 401411.0 & 20036 & 49 & \\
\hline & 19 & 103130.6 & 401451.3 & 19900 & 38 & \\
\hline \multirow[t]{4}{*}{$\overline{\mathrm{A} 1045}$} & 1 & 103500.2 & 304137.5 & 41164 & 182 & \\
\hline & 2 & 103452.2 & 304140.4 & 39408 & 142 & \\
\hline & 3 & 103509.3 & 304603.1 & 40507 & 215 & \\
\hline & 4 & 103500.9 & 3045550 & 36072 & 145 & \\
\hline \multirow[t]{4}{*}{$\overline{\mathrm{A} 1126}$} & 1 & 105402.7 & 165127.6 & 25516 & 61 & \\
\hline & 2 & 105356.3 & $1651 \quad 13.2$ & & & \\
\hline & 3 & 105350.2 & 165100.4 & 25735 & 186 & \\
\hline & 4 & 105345.3 & 165043.3 & & & \\
\hline \multirow[t]{8}{*}{ A1413 } & 1 & 115437.6 & 231250.6 & 42693 & 48 & \\
\hline & 2 & 115537.6 & 231414.1 & 29708 & 42 & \\
\hline & 3 & 115458.4 & 232519.4 & 39846 & 83 & \\
\hline & 4 & 115514.9 & 232753.6 & 45886 & 74 & \\
\hline & 5 & 115500.5 & 232217.2 & 33341 & 76 & \\
\hline & 6 & 115509.0 & 232624.3 & 42913 & 65 & \\
\hline & 7 & 115513.9 & 232848.1 & 29398 & 136 & \\
\hline & 8 & 115535.9 & 231301.3 & 29825 & 78 & \\
\hline \multirow[t]{7}{*}{$\overline{\mathrm{A} 1468}$} & 1 & 120524.9 & 512007.0 & & & \\
\hline & 2 & 120526.5 & 512022.4 & 25590 & 125 & \\
\hline & 3 & 120537.1 & 512141.6 & 24452 & 69 & \\
\hline & 4 & 120528.3 & 512408.7 & 25249 & 54 & \\
\hline & 5 & 120524.2 & 512447.5 & 25288 & 69 & \\
\hline & 6 & 120528.5 & 512847.6 & 25589 & 51 & \\
\hline & 7 & 120551.3 & 512801.9 & 25596 & 52 & \\
\hline \multirow[t]{7}{*}{$\overline{\mathrm{A} 1781}$} & 1 & 134159.7 & 294653.2 & 13699 & 126 & \\
\hline & 2 & 134452.6 & 294616.9 & 18094 & 141 & \\
\hline & 3 & 134414.1 & $2948 \quad 14.8$ & 17577 & 117 & \\
\hline & 4 & 134500.0 & 294855.0 & 17394 & 147 & \\
\hline & 5 & 134411.6 & 293459.1 & 15308 & 66 & \\
\hline & 6 & 134405.3 & 295403.6 & 18909 & 126 & \\
\hline & 7 & 134419.2 & 295559.2 & 11940 & 91 & \\
\hline
\end{tabular}


Table 1. continued

\begin{tabular}{|c|c|c|c|c|c|c|}
\hline CLUSTER & NUMBER & $\begin{array}{l}\text { R.A. } \\
(2000)\end{array}$ & $\begin{array}{l}\text { DEC. } \\
(2000) \\
\end{array}$ & $\begin{array}{l}\text { HEL. VEL. } \\
V\left(\mathrm{~km} \mathrm{~s}^{-1}\right) \\
\end{array}$ & $\begin{array}{l}\text { ERROR } \\
\Delta V\left(\mathrm{~km} \mathrm{~s}^{-1}\right) \\
\end{array}$ & $\begin{array}{l}\text { Other measurements } \\
V\left(\mathrm{~km} \mathrm{~s}^{-1}\right)\end{array}$ \\
\hline \multirow[t]{11}{*}{$\overline{\mathrm{A} 1831}$} & 1 & 135914.9 & 275832.3 & 22762 & 27 & \\
\hline & 2 & 135917.2 & 275606.0 & 17974 & 17 & \\
\hline & 3 & 135908.7 & 280117.5 & 19367 & 14 & \\
\hline & 4 & 135908.8 & 280323.3 & 19701 & 27 & \\
\hline & 5 & 135939.2 & 280339.0 & 10863 & 45 & \\
\hline & 6 & 135936.5 & 280529.7 & 21978 & 32 & \\
\hline & 7 & 135913.5 & 275913.2 & 24078 & 44 & \\
\hline & 8 & 135914.4 & 280055.2 & 24727 & 62 & \\
\hline & 9 & 135908.7 & 275448.1 & 18272 & 33 & \\
\hline & 10 & 135908.2 & 275500.0 & 22568 & 18 & \\
\hline & 11 & 135900.0 & 280401.0 & 18817 & 65 & \\
\hline \multirow[t]{3}{*}{$\overline{\mathrm{A} 2034}$} & 1 & 151011.8 & 332911.0 & 33416 & 50 & $33277 \pm 300$ CEF95 \\
\hline & 2 & 151012.7 & 332932.5 & 33766 & 113 & \\
\hline & 3 & 151013.15 & 332950.2 & 34314 & 118 & \\
\hline \multirow[t]{12}{*}{$\overline{\mathrm{A} 2245}$} & 1 & 170259.7 & 333315.7 & 25336 & 149 & \\
\hline & 2 & 170253.3 & 333400.0 & & & \\
\hline & 3 & 170248.7 & 333424.0 & 26843 & 105 & \\
\hline & 4 & 170245.2 & 333025.2 & 27470 & 78 & \\
\hline & 5 & 170239.3 & 333050.5 & 24553 & 117 & \\
\hline & 6 & 170231.9 & 333120.3 & -107 & 101 & \\
\hline & 7 & 170317.1 & 333243.1 & -146 & 65 & \\
\hline & 8 & 170312.8 & 333240.0 & & & \\
\hline & 9 & 170303.9 & 333234.7 & 18861 & 71 & \\
\hline & 10 & 170217.1 & 332927.4 & 27786 & 70 & \\
\hline & 11 & 170224.6 & 333010.7 & 25508 & 112 & 25452 RK88 \\
\hline & 12 & 170233.1 & 333059.6 & 25895 & 65 & 25932 RK88 \\
\hline \multirow[t]{8}{*}{$\overline{\mathrm{A} 2312}$} & 1 & 185329.5 & $\begin{array}{lll}6823 & 39.8\end{array}$ & 27091 & 93 & \\
\hline & 2 & 185340.6 & 682328.4 & 26882 & 62 & \\
\hline & 3 & 185406.4 & 682255.3 & 28325 & 56 & \\
\hline & 4 & 185415.6 & 682244.1 & -92 & 51 & \\
\hline & 5 & 185345.5 & 682358.7 & 29394 & 42 & \\
\hline & 6 & 185407.3 & 682056.4 & 26229 & 66 & \\
\hline & 7 & 185328.7 & 682834.1 & 7499 & 72 & \\
\hline & & & & $7548^{e}$ & $15^{*}$ & \\
\hline \multirow[t]{9}{*}{$\overline{\mathrm{A} 2457}$} & 1 & 223540.8 & 012905.2 & 17447 & 221 & \\
\hline & 2 & 223528.0 & 012814.7 & 17455 & 185 & \\
\hline & 3 & 223615.3 & 012403.5 & 17589 & 83 & \\
\hline & 4 & 223611.0 & 012429.2 & 17732 & 170 & \\
\hline & 5 & 223554.4 & 012912.3 & 16605 & 286 & \\
\hline & 6 & 223548.9 & 012651.3 & 18597 & 286 & \\
\hline & 7 & 223541.9 & 013931.9 & 16888 & 117 & \multirow{3}{*}{17388 SSG 94} \\
\hline & & & & $16962^{e}$ & 100 & \\
\hline & 8 & 223541.3 & 013653.7 & 17226 & 222 & \\
\hline
\end{tabular}

* emission lines [OII], $\mathrm{H}_{\beta}$, [OIII], $\mathrm{H}_{\alpha},[\mathrm{SI}]$.

(Quintana \& Ramirez 1995; Hewett et al. 1995; Watson et al. 1991). Velocity histograms are shown in Fig. 2.

We have applied to our cluster data set the updated ROSTAT package (version 1.2) developed by Beers et al. (1990). We chose the most appropriate estimators according to Beers et al. (1990), taking into account the number of available velocities $N_{v}$. The results are listed in Table 2. In the "tiny case", when $N_{v} \sim 5$, we have used the median $v_{\mathrm{M}}$ and the bi-weight $v_{\mathrm{BI}}$ as location estimators. In the small-intermediate case $\left(10<N_{v}<20\right)$, we used the median $v_{\mathrm{M}}$ and the bi-weight $v_{\mathrm{BI}}$ as estimators of the location, and the canonical standard deviation $S_{\mathrm{SD}}$, the bi-weight $S_{\mathrm{BI}}$, the gapper $S_{\text {gap }}$. The mean estimator of the location has also been listed, although it is known to be a poor estimator in the case of a non-Gaussian distribution. Table 2 lists in Col. (1), the Abell cluster 
Table 2. Estimates of the redshift and velocity dispersion of the clusters

\begin{tabular}{|c|c|c|c|c|c|c|c|c|c|c|c|}
\hline CLUSTER & $\begin{array}{l}\text { R.A. } \\
(2000) \\
\end{array}$ & $\begin{array}{l}\text { DEC. } \\
(2000)\end{array}$ & $z_{\mathrm{BI}}$ & $z_{\mu}$ & $z_{\mathrm{M}}$ & $z_{\text {previous }}$ & Ref. & $S_{\mathrm{SD}}$ & $S_{\mathrm{BI}}$ & $S_{\text {gap }}$ & $N_{v}$ \\
\hline A195 & 012654 & 191033 & 0.0429 & 0.0431 & 0.0428 & $0.0422 \quad(3)$ & SR87 & 530 & 530 & 540 & 11 \\
\hline A1035 (a) & 103207 & 401233 & 0.0669 & 0.0671 & 0.0669 & & & $(280)$ & $(270)$ & $(280)$ & 9 \\
\hline (b) & & & 0.0785 & 0.0782 & 0.0792 & 0.0799 (1) & SR87 & 860 & 890 & 880 & 10 \\
\hline A1045 & 103500 & 304128 & 0.1351 & 0.1328 & 0.1351 & $0.1381 \quad(1)$ & AEF92 & & & & 5 \\
\hline A1413 & 115522 & 232218 & 0.1424 & 0.1418 & 0.1424 & 0.1427 & SR87 & $(1460)$ & $(370)$ & $(1410)$ & 9 \\
\hline A1468 & 120538 & 512518 & 0.0849 & 0.0844 & 0.0849 & $0.0844 \quad(2)$ & SR87 & & & & 6 \\
\hline A1831 (a) & 135910 & 275928 & 0.0620 & 0.0617 & 0.0614 & $0.0613(11)$ & SR91 & 730 & 670 & 710 & 18 \\
\hline (b) & & & 0.0764 & 0.0764 & 0.0760 & 0.0733 & SR91 & $(790)$ & $(820)$ & $(860)$ & 7 \\
\hline A2034 & 151013 & 333140 & 0.1134 & 0.1134 & 0.1135 & 0.151 & EPS92 & & & & 4 \\
\hline A 2245 & 170244 & 333148 & 0.0862 & 0.0864 & 0.0852 & 0.085 & RK88 & $(1100)$ & $(1160)$ & $(1150)$ & 9 \\
\hline A2312 & 185338 & 682153 & 0.0918 & 0.0920 & 0.0905 & 0.0928 & PHG92 & & & & 5 \\
\hline A 2457 & 223545 & 012833 & 0.0582 & 0.0580 & 0.0582 & $0.0585 \quad(1)$ & QR95 & 550 & 420 & 530 & 14 \\
\hline
\end{tabular}
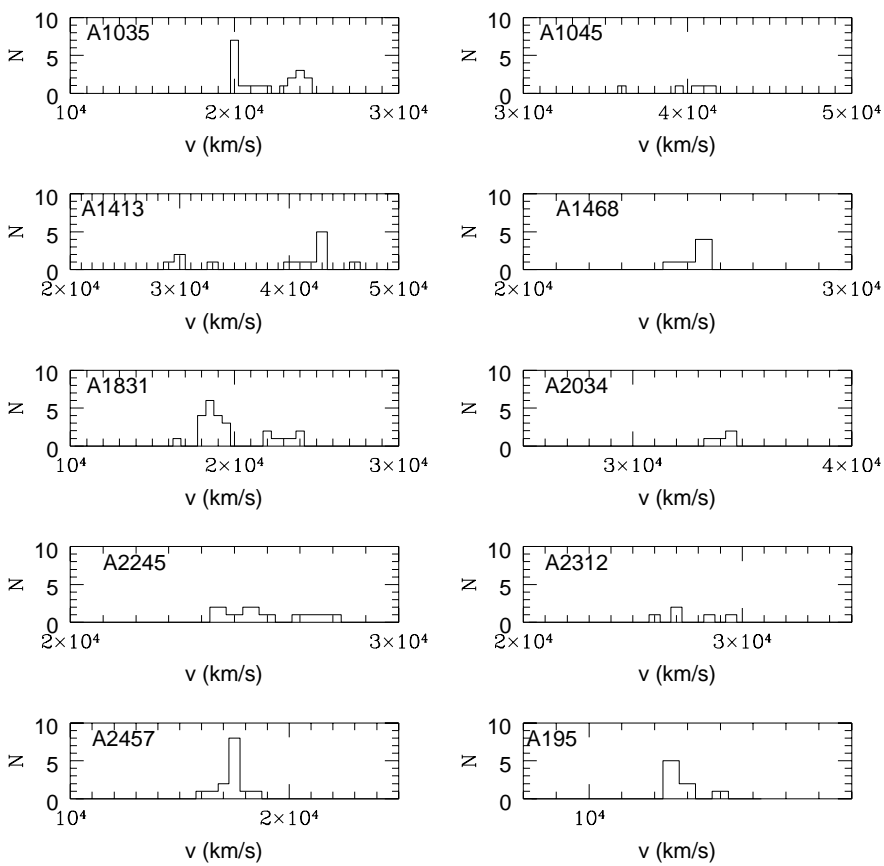

Fig. 2. The line-of-sight velocity histograms for our target clusters

number, in Col. (2) and (3) the right ascension and declination of the cluster center (Abell, et al. 1989) precessed at J2000.0, in Cols. 4, 5 and 6, bi-weight, median and mean estimates of the redshift of the cluster from our analysis, in Col. 7 the previous measurement of the cluster redshift from literature (referenced in Col. 10) and the number of galaxies used for this estimate, in Cols. 11, 12 and 13, the standard deviation, bi-weight, and gap estimates of the velocity dispersion within the cluster, and in Col. 13 the number of cluster members used for the previous determinations. The velocity analysis is indeed limited by the small number of galaxies with available redshift by cluster. The various estimates of velocity dispersions are listed in
Table 2 so that the degree of self-consistency can easily be checked. Determinations for clusters with less that 10 redshift measured are listed between braces.

\section{Note on individual clusters}

Some clusters of our sub-sample show a complex velocity distribution. For instance, it is clear from Fig. 2 that the velocity distribution of $\mathbf{A 1 0 3 5}$ is bimodal, with a group of 9 galaxies clustered around $v_{\mathrm{BI}}=$ $20052 \mathrm{~km} \mathrm{~s}^{-1}$ with small dispersion $\left(\sigma \sim 280 \mathrm{~km} \mathrm{~s}^{-1}\right)$ and a group of 10 galaxies belonging a to a more massive structure around $v_{\mathrm{BI}}=23530 \mathrm{~km} \mathrm{~s}^{-1}\left(\sigma \sim 880 \mathrm{~km} \mathrm{~s}^{-1}\right)$.

A1781 shows a complex structure that cannot be well sampled with our data. The field of this cluster was recently examined in detail by Ramella et al. (1995; RGHT), as a loose group of galaxies at $z \sim 0.04$ is found in that region of the sky. This results in a projection of the loose group on the more distant Abell cluster (at $z \sim 0.065$ from Postman et al. 1992), of which galaxies $2,3,4$, and 6 are probably genuine members. In Fig. 3), the chain of four galaxies in the north corresponds to a foreground structure, the redshifts of these galaxies being respectively, from south to north, $11019 \mathrm{~km} \mathrm{~s}^{-1}$ (RGHT), $13699 \mathrm{~km} \mathrm{~s}^{-1}$ (our galaxy No.1), $11928 \mathrm{~km} \mathrm{~s}^{-1}$ (RGHT), and $11940 \mathrm{~km} \mathrm{~s}^{-1}$ (our galaxy No. 7).

A1831 shows two peaks in the velocity histogram, at $v_{\mathrm{BI}}=18585 \mathrm{~km} \mathrm{~s}^{-1}$ and $v_{\mathrm{BI}}=22907 \mathrm{~km} \mathrm{~s}^{-1}$, with velocity dispersions of respectively $\sim 700 \mathrm{~km} \mathrm{~s}^{-1}$ and $\sim 820 \mathrm{~km} \mathrm{~s}^{-1}$. As mentioned before, we will not pursue the analysis of the three previous clusters.

A1413 shows a main concentration of 9 galaxies at a mean velocity of $v_{\mathrm{BI}}=42686 \mathrm{~km} \mathrm{~s}^{-1}$ (including the central cD galaxy) identified as the main cluster. The estimate of its velocity dispersion (listed in Table 2) is $S_{\mathrm{SD}}=$ $1460 \mathrm{~km} \mathrm{~s}^{-1}$. This value is quite different from the estimate with the bi-weighted method $\left(S_{\mathrm{BI}}=370 \mathrm{~km} \mathrm{~s}^{-1}\right)$. This can mean that the number of galaxies is still not sufficient to use the bi-weighted technique. Previous estimates 
of the velocity dispersion in A1413 have to be taken with care until more redshift measurements are available.

Finally, we note the presence of a foreground group of 4 galaxies around $v=30000 \mathrm{~km} \mathrm{~s}^{-1}$.

In A2457, the spectrum of galaxy 7 (Fig. 3) shows three very broad systems of emission lines. The first complex includes a blend of $\mathrm{H}_{\gamma}$ and [O III], with a FWHM of about $89 \AA$, the second one (with a FWHM of $\sim 140 \AA$ ) includes $\mathrm{H} \beta$ and the [OIII] doublet in the tail of $\mathrm{H} \beta$, the third one (FWHM $\sim 115 \AA$ ) includes a blend of $\mathrm{H}_{\alpha}$ and $[\mathrm{N}$ II]. Accurate fits with a gaussian could be done when deblending the [OIII] doublet and the $\mathrm{H}_{\alpha}$ and [N II] components, giving a mean velocity of $v=16962 \pm 100 \mathrm{~km} \mathrm{~s}^{-1}$. The cross-correlation technique for the absorption lines gives $v=16888 \pm 117 \mathrm{~km} \mathrm{~s}^{-1}$. This object has been included for its $\mathrm{H}_{\alpha}$ and [SII] lines in the catalog of the Palomar Transit Grism Survey (Schneider et al. 1994), which gives a velocity $v=17388 \pm 300 \mathrm{~km} \mathrm{~s}^{-1}$. The difference with our estimate is easily explained by the fact that in the case of the PTGS $\mathrm{H}_{\alpha}$ and [N II] could not be deblended, given the low-resolution of that survey; as a consequence, the wavelength of the $\mathrm{H}_{\alpha}$ line -and the redshift- is overestimated.

The existence of very broad permitted emission lines and narrower forbidden ones suggests that this object is a Seyfert 1 galaxy.

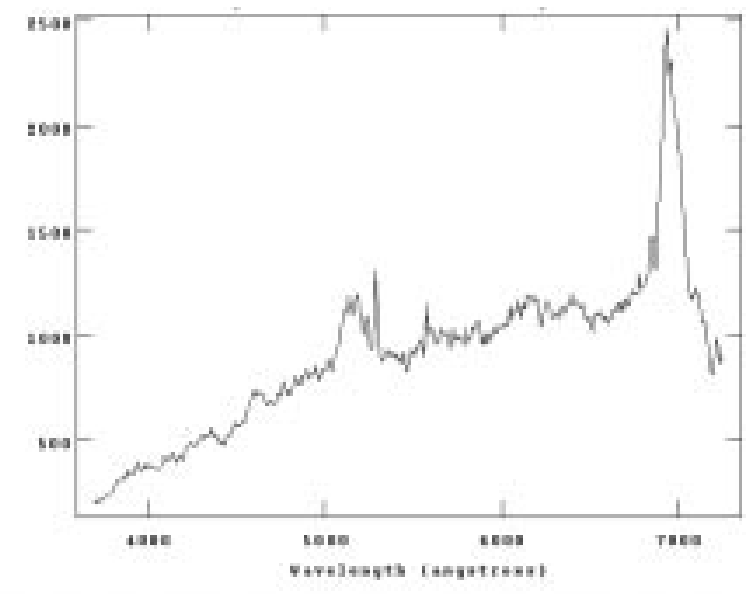

Fig. 3. The sky-subtracted spectrum of galaxy 7 in A2457. This object shows emission lines characteristic of a Seyfert I galaxy

\section{Conclusion}

We have presented the first results of an observational program devoted to the measure of cluster redshifts and velocity dispersions. At the present stage of this work, estimates of the velocity dispersions have to be considered as preliminary results as a consequence of the small number of measured redshifts per cluster. As a second step, we are carrying on further spectroscopical observations with multi-object facilities in order to increase efficiently the data for each cluster. The information which can be derived from these data is of course useful for various studies, from large-scale structure to cluster dynamics. Our main aim is to use this database to constrain the parameters defining the cluster fundamental plane.

Acknowledgements. We would like to thank all the staff of the Observatoire de Haute Provence for their efficient assistance during the successive observational runs. S.M. would like to thank Véronique Cayatte, Catherine Boisson and Philippe Prugniel for fruitful discussions. We are grateful to Jean Guibert and the MAMA team for their scans of the POSSII plates. This research has made use of the NASA/IPAC extragalactic database (NED) which is operated by the Jet Propulsion Laboratory, Caltech, under contract with the National Aeronautics and Space Administration.

\section{References}

Abell G.O., Corwin H.G., Olowin R.P., 1989, ApJS 70, 1

Allen S.W., Edge A.C., Fabian A.C., et al., 1992, MNRAS 259, 67

Beers T., Flynn K., Gebhart K., 1990, AJ 100, 32

Batuski D.J., Burns J.O., Newberry M.V., et al., 1991, AJ 101, 1983

Cole S., 1989, PhD Thesis

Crawford C.S., Edge A.C., Fabian A.C., et al., 1995, MNRAS 274,75

Danese L., de Zotti G., di Tullio G., 1980, A\&A 82, 322

Dressler A., Lyndel-Bell D., Burstein D., et al., 1987, ApJ 313, 42

Djorgovski G., Davis M., 1987, ApJ 313, 59

Elvis M., Plummer D., Schachter J., Fabbiano G., 1992, ApJS 80,257

Giovanelli R., Haynes M.P., 1993, AJ 105, 1271

Hewett P.C., Foltz C.B., Chaffee F.H., 1995, AJ 109, 1498

Le Fèvre O., Crampton C., Lilly S.J., Hammer F., Tresse L., 1995, ApJ 455, 60

Lemaitre G., et al., 1990, A\&A 228, 546

Owen F., Ledlow M., Keel W., 1995, AJ 109, 140

Postman M., Huchra J., Geller M., 1992, ApJ 384, 404

Quintana H., Ramirez A., 1995, ApJS 96, 343

Ramella M., Geller M.J., Huchra J.P., Thorstensen J.R., 1995, AJ 109, 1458

Rhee G., Katgert P., 1988, A\&AS 72, 243

Schaeffer R., Maurogordato S., Cappi A., Bernardeau F, 1993, MNRAS 263, L21 (SMCB)

Schneider D., Schmidt M., Gunn J., 1994, AJ 107, 1245

Slezak E., Bijaoui A., Mars G., 1988, A\&A 201, 9

Stocke J.T., Morris S.L., Gioia I.M., et al., 1991, ApJS 76, 813

Struble M.F., Rood, H.J., 1987, ApJS, 63, 543

Struble M.F., Rood, H.J., 1991, ApJS 77, 363

Tonry J., Davis, M., 1981, ApJ 84, 1511

Watson F.G., Oates A.P., Shanks T., Hale-Sutton D., 1991, MNRAS 253, 222

West M.J., Dekel A., Oemler A., 1987, ApJ 316, 1

West M.J., Oemler A., Dekel A., 1989, ApJ 346, 539

Zabludoff A.I., Huchra J.P., Geller M.J., Vogeley M.S., 1993, AJ 106, 1273 